\title{
Revealing the details of vortex core precession in cyclones by means of large-eddy simulation
}

\author{
Lakhbir Singh Brar ${ }^{1}$ \\ Mechanical Engineering Department, Birla Institute of Technology, Mesra, Ranchi 835215, India
}

J.J. Derksen

School of Engineering, University of Aberdeen, Aberdeen, UK

${ }^{1}$ E-mail address of the corresponding author: brarlsb@gmail.com, brarls@bitmesra.ac.in

\section{Chemical Engineering Research \& Design - Accepted April 2020}

\begin{abstract}
We investigate the three-dimensional, time-dependent behaviour of coherent flow structures inside two cyclone separator geometries, a Stairmand cyclone and a swirl tube, operating in the turbulent regime. For this, large-eddy simulations (LES) with a standard Smagorinsky subgrid-scale model are carried out on the single-phase flow employing a nonorthogonal curvilinear hexahedral mesh. Reynolds numbers $(R e)$ are in the range $9 \cdot 10^{4}$ to $4 \cdot 10^{5}$. The simulations successfully capture the quasi-periodic precessing vortex core (PVC) phenomenon. The spectra derived from the temporal signals of different flow variables viz. static pressure, tangential velocity, axial velocity and radial velocity in the Stairmand cyclone exhibit two distinct frequencies at all $R e$, whereas the spectra for the swirl tube show a single peak. These spectra strongly resemble those reported in the experimental literature. The periodicity pertaining to the inner vortex about its rotational axis is also analysed. Next, the effect of $R e$ on the two distinct spectral peaks have been evaluated for the Stairmand cyclone. Lastly, we present the effect of $R e$ on mean and fluctuating fields and their variations along the geometrical axis of the cyclone.
\end{abstract}

Key words: Cyclone separators; Large eddy simulation (LES); Precessing vortex core (PVC); Pressure fluctuations; Velocity fluctuations 


\section{Introduction}

Confined vortex flow finds significance in numerous industrial processes. Such flows are used for mixing (Levy and Albagli, 1991; Liu et al., 2017) as well as for the separation of two or more phases with different densities (Sommerfeld and Qiu, 1993), and also for flow stabilisation (as in swirl burners (Froud et al., 1995)). One popular application of swirldominated flow and the subject of this study is the cyclone separator. Cyclone separators are devices that utilise net centrifugal forces generated by swirling fluid motion to separate phases with different densities. As a result of the higher density of the solids compared to the gas, the particles experience a net centrifugal force that drives them towards the outer wall of the cyclone. Once caught in the wall boundary layer, the particles spiral downward along the wall under the influence of net gravity and (more so) by an on average downward velocity in the near-wall region, until they enter a collection bin.

Typically, in reverse flow cyclones, the primary flow constitutes double vortex: the outer (or free) vortex that spirals down, and the inner (or forced) vortex that spirals in the upward direction. The inner vortex exhibits a very complex motion - this coherent structure precesses around the geometrical axis, referred to as the precessing vortex core (PVC) phenomenon. It contributes significantly to velocity fluctuation levels in - given its timeperiodic nature - a non-turbulent manner and gives rise to fluctuations in the core region with a regular frequency and somewhat less regular amplitude (Yazdabadi et al., 1994). In cyclone separators, PVC phenomenon could enhance particle dispersion and cause a reduction in collection efficiency, specifically for situations where the (relaxation) timescales of particles interfere with the timescales associated with precession (i.e. $1 / f$; with $f$ the precession frequency of inner vortex).

The frequency of the precessing motion is affected by the geometry and flow conditions (the flow rate, in particular). In dimensionless form, the former can be expressed in terms of 
swirl number and the latter in terms of the Reynolds number, respectively. The swirl number quantifies the ratio of tangential momentum over axial momentum. For cyclones in which swirl is generated through a tangential inlet, a geometrical swirl number is often used as an estimate of the actual swirl number: $S \equiv \pi D_{e} D / 4 A_{i}$ with $D, D_{e}$ and $A_{i}$ the main body diameter, vortex finder diameter, and cross-sectional area of inlet, respectively (see Fig. 1 for the two flow configurations investigated in this paper). The Reynolds number is usually based on the superficial inlet velocity $U_{\text {in }}$ and the body diameter: $R e \equiv U_{i n} D / v$ with $v$ the kinematic viscosity of the working fluid. The precession frequency is non-dimensionalized by $U_{\text {in }}$ and $D$ to form a Strouhal number: $S t \equiv f D / U_{i n}$. In dimensionless terms we expect $S t$ to be a function of $R e$ and $S$.

Although the history of cyclone separators goes back more than a century (Morse,1886), research is ongoing to describe and understand the complicated flow pattern, and its consequences on the particle separation process. Solero and Coghe (2002) performed experiments on a laboratory scale cyclone separator using laser Doppler anemometry (LDA) with a body diameter of $D=0.288 \mathrm{~m}$ and reported a distinct low-frequency peak with $S t=0.617$ at the volume flow rate of $0.63 \mathrm{~m}^{3} / \mathrm{s}$. Hoekstra (2000) conducted experiments on Stairmand highefficiency cyclone with $D=0.29 \mathrm{~m}$ as well as on a swirl tube with $D=0.10 \mathrm{~m}$. In Stairmand cyclone, at $R e=2.8 \cdot 10^{5}$, two distinct spectral peaks were reported viz. $S t=0.7$ and $1.58-$ the latter harmonic was related to the precessional motion of the inner vortex about the central axis (Derksen, 2003). Spectral estimates from velocity fluctuations in the swirl tube at $R e=0.15 \cdot 10^{5}$ yielded only one distinct spectral peak at $S t=0.49$ - this (nondimensional) frequency was associated with vortex core precession. Gao et al. (2010) reported two distinct peaks from the pressure spectra viz. $S t=1.08$ and $S t=2.31$ at $R e=3.3 \cdot 10^{5}$. Based on the variations in the power of spectral peaks along the radial direction, the first peak was interpreted as the frequency of outer vortex, and the second peak with inner vortex. Gu et al. (2016) performed a similar study and reported two dominating peaks in the pressure spectra viz. $S t=1.07$ and $S t=2.69$ at $R e=$ 
$2.5 \cdot 10^{5}$ for clean air. The second harmonic was reported as the precessing frequency of vortex centre, whereas the first harmonic was related to the quasi-forced vortex rotation. Akiyama and Kato (2017) performed experimental and numerical study to investigate the flow field and collection efficiency on a 70mm diameter cyclone model. Two peaks were derived from the tangential velocity spectra by means of CFD. Jia et al. (2019) performed experiments and reported the outer vortex to induce a low frequency range pressure fluctuation with high magnitude, and the inner vortex to induce a new main frequency due to the periodically rotating flow behaviour. The dominant frequency in the vortex finder of the cyclone separator was related to inner vortex rotation. Gao et al. (2019) performed experiments and observed the flow to develop from single vortex motion (dominated by a concentrated frequency) to a double vortex motion (dominated by two concentrated frequencies).

It becomes apparent from the earlier studies that despite several (mainly experimental) efforts, large discrepancies are observed. For instance, the rotation of inner vortex was related to the first spectral peak by Gu et al. (2016) and Jia et al. (2019) without sufficient evidence this speculation was based on the condition (adopted from other studies) that tangential velocity approaches the inlet velocity at $r / R=0.2$. The operating conditions (especially $R e$ ) and geometry being considerably different from those reported, which could be misleading. Akiyama and Kato (Akiyama and Kato, 2017) wrongly mentioned the findings from the experimental values of Derksen (2003) and based on this, the authors interpreted the lower harmonic as the precessional frequency of inner vortex - no information on the higher harmonic was provided. Therefore, we conduct a computational study to reinvestigate the nature of the precessing coherent structures and their effects on the mean and RMS velocity profiles of different flow behaviour by means of computational fluid dynamics (CFD).

This paper is organized in the following manner: first, the flow systems are defined along with the details on mesh generation, and boundary conditions used in the simulations. 
Second, the validation of simulation methodology against the experimental data is performed at different grid levels and time step size. Third, we analyse the effect of $R e$ on mean and RMS profiles of static pressure as well as velocity components. Lastly, the spectra are derived from temporal signals of different flow variables both in Stairmand cyclone and swirl tube.

\section{Description of the flow geometry and validation}

\subsection{Flow Geometry}

Two different cyclone geometries are investigated, one of which has been used by Hoekstra (2000) while the other closely resembles one of his devices. The first one is a cylinder-on-cone type model with the design guidelines proposed by Stairmand (1951) having the main body diameter, $D=0.29$ m (cf. Fig. 1) - the geometric swirl number $S=3.93$, and a collection bin is adopted in accordance with Hoekstra (2000). The geometry of the second flow model is similar to that of Hoekstra et al. (1998), also used by Derksen and Van den Akker (2000). It has the main body diameter $D=0.10 \mathrm{~m}$ and $S=2.08$ (cf. Fig. 1). In both geometries, the origin of the coordinate system is located on the geometrical axis of the cyclone, over the roof, with $z$-direction pointing downwards as positive, as illustrated in Fig. 1.

\subsection{Turbulence modelling}

Two approaches viz. a second-order closure model for RANS and LES are widely used to predict the cyclonic flows. In the former approach, due to the averaging of $N S$ equations over a time frame much longer than the period of fluctuation of the flow parameters - the velocity fluctuations (an important component of the instantaneous velocity) are lost. The latter approach, on the other hand, works on a filtering method where large scales are separated from the smaller ones (Ferziger and Perić, 2002). In Fluent v16.2, which is the software that we used to perform all simulations in this paper, the filtering operation is performed by the grids itself. 
Eddies with sizes larger than grid size are resolved explicitly and do not require any modelling, whereas the eddies that are smaller than the grid size are modelled using the sub-grid scale (SGS) model. The unknown SGS stresses resulting from the filtering operation are modelled using the Boussinesq hypothesis (Hinze, 1975), which computes SGS stresses as:

$$
\tau_{i j}-\frac{1}{3} \tau_{k k} \delta_{i j}=-2 \mu_{s g s} \bar{S}_{i j}
$$

where, $\tau_{i j}$ is the SGS stress tensor, $\mu_{s g s}$ is SGS turbulent viscosity, $\delta_{i j}$ is the Kronecker delta, and $\bar{S}_{i j}$ is the rate-of-strain tensor associated to the resolved velocity field $\bar{u}_{i}$. It is defined as:

$$
\bar{S}_{i j}=\frac{1}{2}\left(\frac{\partial \bar{u}_{i}}{\partial x_{j}}+\frac{\partial \bar{u}_{j}}{\partial x_{i}}\right)
$$

In Eq. (1), the isotropic part of the subgrid-scale stresses, $\tau_{k k}$, is not modelled, but added to the filtered static pressure term (Ferziger and Perić, 2002).

For turbulence modelling, the standard Smagorinsky SGS model (Smagorinsky, 1963) has been employed - the subgrid scale viscosity is calculated as:

$$
\mu_{s g s}=\rho l_{m i x}^{2}\left|\bar{S}_{i j}\right|
$$

where $\rho$ is the fluid density, $l_{m i x}$ is the mixing length for subgrid scales, and $\left|\bar{S}_{i j}\right|=\sqrt{2 \bar{S}_{i j} \bar{S}_{i j}}$ is the resolved deformation rate (Ferziger and Perić, 2002). The local filter width is related to the local grid size and wall distance as:

$$
l_{\text {mix }}=\min \left(\kappa d, C_{S} \Delta\right)
$$

where $\kappa$ is the von Kármán constant that has been taken as $0.41, d$ is the distance to the closest wall, and $\Delta$ is a measure for the local grid spacing computed as $\Delta=\mathrm{V}^{1 / 3}$ (with $\mathrm{V}$ the local cell volume). In the present study, we use the Smagorinsky constant, $C_{S}=0.1$. Earlier studies have demonstrated its utility to accurately resolve the 3-D, time-dependent flow including PVC phenomenon, and predicting the mean and RMS velocity values (e.g.: Derksen and Van den 
Akker, 2000; Derksen, 2003; Gronald and Derksen, 2011; de Souza et al., 2012; Dhakal et al., 2014; Brar and Elsayed, 2017).

\subsection{Discretization of the solution domain}

Three-dimensional cyclone models were built to the scale, as in the experimental studies, using SolidWorks 2014 (cf. Fig. 1). The model was imported into ICEM CFD v16.2 to generate the block-structured mesh. A single block was created first and then split down into several parts to fill the entire flow domain. Such decomposition of blocks facilitates in generating the hexahedral mesh with full control over the global/local grid refinement. The boundary-fitted mesh is generated that gets easily adapted to the geometry. Since the grid lines do not intersect orthogonally, the mesh is non-orthogonal, boundary-fitted in nature. The control volumes (cells) in the fluid region have been aligned with the flow direction to ensure least numerical diffusion (Wendt, 2008).

For validation, three levels of grid viz. coarse mesh consisting of about 0.7 million hexahedra, medium mesh consisting of around 1.8 million hexahedra, and fine mesh consisting of nearly 2.9 million hexahedra have been created. The mesh density is increased by a factor of 1.35 and 1.6 (based on the coarse mesh) in each of the three coordinate directions for generating the medium and fine mesh, respectively. Surface mesh consisting of 0.7 million hexahedra (to the left) and 2.9 million hexahedra (to the right) of Stairmand cyclone is illustrated in Fig. 2.

For the swirl tube, a similar technique of building grid was employed. Two levels of grid viz. coarse mesh consisting of 0.6 million hexahedra and fine mesh (with density increased by a factor of 1.4 in each coordinate direction) of 1.8 million hexahedra, were used.

\subsection{Near-wall treatment}


The large velocity gradients in the wall-normal direction demand special treatment of boundary layer flow while performing numerical simulations. In the present study, owing to the coarse mesh in near-wall region, the wall adjacent first cell centroid is in turbulent zone, to which the solver applies the law-of-the-wall approach (Gronald and Derksen, 2011; de Souza et al., 2012; Shukla et al., 2013; Brar and Elsayed, 2017). At the wall, the no-slip condition - a Dirichlet boundary condition - is imposed. With classical Smagorinsky model, since the eddy viscosity does not get adapted to the proximity of wall, a wall damping function is required to explicitly reduce the eddy-viscosity to zero in that region. For this, one of the first mixing length models proposed by Prandtl (1925), as represented by the first term in the parenthesis of Eq. 4, is utilized to make the eddy viscosity go to zero at the wall (cf. Eq. 3), thus accounting for the near-wall damping.

\subsection{Boundary conditions}

The numerical simulations were carried out with air as the working medium having kinematic viscosity $1.667 \cdot 10^{-5} \mathrm{~m}^{2} / \mathrm{s}$, temperature $293 \mathrm{~K}$, pressure 1.0 atm. and a density of 1.20 $\mathrm{kg} / \mathrm{m}^{3}$.

The velocity inlet boundary condition with zero-gauge pressure was used. The air was introduced in face normal direction into the cyclone with a presumed uniform velocity profile. For Stairmand cyclone, five different values of $R e$ viz. $0.87 \cdot 10^{5}, 1.74 \cdot 10^{5}, 2.80 \cdot 10^{5}, 3.48 \cdot 10^{5}$ and $4.35 \cdot 10^{5}$ that corresponds to the superficial inlet velocity of $5.0 \mathrm{~m} / \mathrm{s}, 10.0 \mathrm{~m} / \mathrm{s}, 16.1 \mathrm{~m} / \mathrm{s}, 20.0$ $\mathrm{m} / \mathrm{s}$, and $25.0 \mathrm{~m} / \mathrm{s}$, respectively, have been used. The simulation of swirl tube is performed at $R e=0.90 \cdot 10^{5}$.

Under the sub-critical state of swirling fluid, small perturbations are likely to propagate downstream and upstream, whereas under supercritical state the perturbation only propagates downstream (Alekseenko et al., 2007). Due to (possible) sub-critical behaviour by the strong 
swirling flow that approaches the outlet boundary, a special treatment is required there. As in many earlier studies that have reported reasonably good agreement with the experimental data (e.g.: de Souza et al., 2012; Dhakal et al., 2014; Brar and Elsayed, 2017; Brar and Elsayed, 2018), we use pressure outlet boundary condition at the outlet surface with zero-gauge pressure and the normal to boundary method to specify the backflow (de Souza et al., 2012; Brar and Elsayed, 2018). The rest of the boundaries were defined as walls with a no-slip condition and additional wall treatment as discussed earlier in the section on near-wall treatment.

\subsection{Simulation methodology}

The numerical simulations were performed using both steady as well as transient conditions. To begin with, steady-state simulations using RNG $k-\varepsilon$ model were carried out to generate an initial flow field. The resulting field was initialized using a text user interface (TUI) command "init-instantaneous-vel" to generate a realistic initial field for LES start-up - this considerably reduced time and computational effort.

Since the flow inside the cyclone separators is almost incompressible, the pressurebased implicit solver is preferred [31]. The PRESTO! (PREssure STaggering Option) scheme is applied to interpolate the pressure values at the cell faces. This scheme is similar to the staggered grid for structured meshes (Patankar, 2018) and is suitable for flows in strongly curved domains and high swirl intensity. Pressure and velocity have been coupled using the fractional-step method (FSM) - originally introduced by Chorin (1968) and Temam (1969) which is available only with the non-iterative time-advancement (NITA) algorithm. The diffusion terms in momentum-transport equations are discretized using the second order bounded central differencing scheme. The cell gradients are evaluated using the least-squares cell-based method. For time-advancement, the bounded second-order accurate implicit method was adopted. Instead of using the iterative time-advancement scheme - in which a large number 
of outer iterations performed for each time-step - NITA scheme was preferred that saves a considerable amount of time, although it consumes a larger memory (Brar and Elsayed, 2017); Brar and Elsayed, 2017). NITA preserves the overall time accuracy by making the splitting error of the same order as the truncation error rather than reducing it to zero.

All the numerical simulations were performed on a PC with Intel i7-3930K (a 6-core) processor operating at a base frequency of $3.20 \mathrm{GHz}$ (and a turbo frequency of $3.8 \mathrm{GHz}$ ), $16 \mathrm{~GB}$ RAM and 2TB HDD. The simulations performed on Stairmand cyclone at $R e=2.80 \cdot 10^{5}$ elucidated that the physical run-time to carry out a single-phase simulation on the coarse mesh, medium mesh, and fine mesh was nearly one and a half-day, four days, and nine days, respectively. Over this physical time, the corresponding flow time was $138.5 T_{\text {in }}$ (with $T_{\text {in }}=D / U_{\text {in }}$ the integral time scale).

\subsection{Validation}

To validate the simulation methodology, Stairmand high-efficiency cyclone (cf. Fig. 1(a)) with $S=3.93$, operating at $R e=2.8 \cdot 10^{5}$ (based on superficial inlet velocity of $16.1 \mathrm{~m} / \mathrm{s}$ ) is adapted from the experimental study (Hoekstra, 2000). By traversing the laser Doppler anemometry (LDA) measuring volume along the radial direction, the axial and tangential velocities were measured. The mean and root-mean-square velocity values were determined from the LDA data sets, and the precessing frequency of the coherent structure was also presented.

The time-independent simulations were started from a zero-velocity field using RNG $k-\varepsilon$ model for 2000 iterations at $R e=2.8 \cdot 10^{5}$. The LES simulations with prescribed solver settings were performed on the resulting field for $t U_{i n} / D=38$ before starting the time averaging operation. It was also ensured that the monitored tangential velocity at various locations had become quasi-steady. A similar procedure has been adopted for all the test models. 
The first column in Fig. 3 illustrates the mean tangential velocity profiles predicted using LES at $Z / D=0.75,2.0$ and 2.5 that fits well to the available data (with a slightly increased slope in the core region at all levels of the grid). At $Z / D=2.0$ and 2.5 , the LES predictions mildly overestimate the peaks of tangential velocity. Also, with grid refinement, no significant differences are observed, except in the forced vortex region, where a small deviation in the (linear variation of) tangential velocity profile is observed with the finest mesh. The second column in Fig. 4 shows the mean axial velocity profiles that agree qualitatively both in the free and the forced vortex regions. However, at $Z / D=2.5$, the numerical predictions are closer to being symmetric than the actual profile. The dip near the cyclone axis, is well predicted by LES. Also, the axial velocity profiles are virtually identical at all three levels of grid resolution.

The RMS velocity profiles are more complicated than their respective mean velocity profiles. Increased levels of velocity fluctuations are observed in the forced vortex region. The first column in the right-side block of Fig. 3 presents the LES prediction for RMS tangential velocity compared to the experimental data. The LES predictions in the outer vortex agree well. A mismatch is observed in the core region, where instead of exhibiting a dip, a rise in RMS values (particularly at $Z / D=0.75$ and 2.0) is observed that further increases with grid refinement. The second column in the right-side block of Fig. 3 illustrates a comparison of the LES predicted RMS axial velocity profiles with the LDA data at the three axial locations. The results agree qualitatively in both the forced and free vortex zones. The mesh refinement could have led to the small errors (e.g. in the average velocity profiles) getting amplified in the precession-induced fluctuation levels. However, with the known issues to resolve the flow anisotropy using LES (de Souza et al., 2012), the above results, provide a detailed assessment of the levels of accuracy of our simulation procedure.

The vortex core precession was measured by Hoekstra (2000) (and presented by Derksen (2003)) at $Z / D=2.0$ where the velocity-time series was captured by means of LDA. 
Two distinct peaks were reported in the power spectral density (PSD) plot at $R e=2.80 \cdot 10^{5}-$ the first harmonic peak at $S t=0.70$ and the second peak at $S t=1.58$. The latter peak is the principal frequency of the flow system (Derksen, 2003). Fig. 4 presents the PSD plot derived from the fast Fourier transformation (FFT) of $\mathrm{Y}$ velocity-time series data recorded at $X / D=Y / D=Z / D=0$ for different levels of the grid. The difference in the first and second harmonic peaks are within $3 \%$ and $2 \%$, respectively. Therefore, a good agreement between the LES predictions at all levels of the grid is observed. In addition, our simulation results are within $10 \%$ of the experimental data for the Strouhal numbers. The dependency of the time step size on the PVC

inside Stairmand cyclone has also been tested at $R e=2.80 \cdot 10^{5}$ (cf. Fig. 4). At $\Delta t / T_{i n}=5.56 \cdot 10^{-3}$ and $2.78 \cdot 10^{-3}$, St for lower harmonics are both equal to 0.670 , whereas the higher harmonics correspond to 1.495 and 1.499 , respectively. The above results indicate no dependency of the simulation results on the time step sizes - thus, $\Delta t / T_{i n}=5.56 \cdot 10^{-3}$ was adopted for the simulation of Stairmand cyclone model for all Re. The two different time steps resulted in the cell convective Courant numbers of approximately 16 and 8, respectively. Since the solver used is segregated implicit, there are no limitations on the stability based on the Courant number (Elsayed and Lacor, 2011).

\section{Results and discussion}

The effect of PVC - together with the gradients from the mean profiles of velocity and pressure fields - result in high fluctuation levels of velocity components and pressure in core region. Therefore, in this section the dynamic behaviour and periodic nature of such coherent structure, and its effect on the flow field is explored.

\subsection{Path followed by vortex core}


The offset centre of the rotating vortex from the geometrical axis in Stairmand cyclone is evaluated first. For this, the realizations based on instantaneous static pressure are extracted on a horizontal plane inside the vortex finder tube (cf. Fig. 5 (1-20)) close to the cyclone roof $(Z / D=0)$. A small white spot near the geometrical axis - that corresponds to the point of least pressure - can be associated with the core of the vortex. Time spacing between each realization is $0.067 \mathrm{D} / U_{\text {in }}$ - this time gap is nearly one-tenth of the time vortex core takes to precess about the cyclone axis. Throughout the motion, the vortex centre avoids the geometrical centre. More detailed information on the movement of vortex core centre over a horizontal plane $Z / D=0.0$ is presented in Fig. 6(a). The data have been recorded at each time step for a duration of $17.21 \mathrm{D} / U_{i n}$, while starting at $35.23 \mathrm{D} / U_{i n}$. The path traced out by the vortex centre is seemingly erratic. Based on the $x$ - and $y$-positions of the vortex core centre (from Fig. 6(a)) the probability density function (PDF) are calculated. The first moment equals $0.0124 R$ with standard deviation of $0.033 R$ (cf. Fig. 6(b)) for $x$-position and the first moment of $0.012 R$ with standard deviation of $0.042 R$ (cf. Fig. 6(c)) for $y$-position, respectively. PDF based on radial locations (calculated from $x$ - and $y$-positions) also illustrates the offset vortex core from the geometrical axis with the first moment located at $0.045 R$ and standard deviation of $0.023 R$ (cf. Fig. 8(d)). As presented in Fig. 6(e), the vortex core has preferred angular location close to $3 \pi / 2$ (with actual value of $4.256 \mathrm{rad})$.

The above analyses were confined to the planar motion of the offset vortex core centre from the geometrical centre. This can be extended to a three-dimensional representation over the entire cyclone length. Fig. 6(f) presents the average distance of the rotational axis of the vortex core from the geometrical axis in Stairmand cyclone. The radial distance is scaled up by a factor of 5 in both $x$-and $y$-directions (with no scaling in the $z$-direction) for better visibility. The rotational axis of the vortex core takes up a helical path around the geometrical axis and 
resembles a twisted rope-like structure. The offset is more prominent both in the separation space and inside collection bin than inside the vortex finder.

\subsection{Vortex-core precession}

The PVC is an internally driven mechanism that exhibits irregularities, namely, phase jump and irregular amplitude. Furthermore, the velocity fluctuations monitored at a point, over time, are the superposition of turbulent fluctuations onto the periodic signals. Hence, the individual contribution of coherent fluctuations and turbulent fluctuations in the main flow are hard to define. However, the periodicities, such as due coherent fluctuations, could be calculated by performing FFT of the fluctuating signals in which the complex, time-dependent nonlinear signals are transformed from the time domain to the frequency domain. In this section, we present the spectral estimates derived from the temporal signals of flow variables such as static pressure, tangential velocity, axial velocity and radial velocity both in Stairmand cyclone and swirl tube.

The dimensionless frequency values are estimated inside the vortex finder tube of Stairmand cyclone model at radial positions $X / D=-0.075,-0.150$ and -0.225 (cf. Fig. 7). The spectrum derived from static pressure fluctuations represents two harmonics at locations $X / D=-$ 0.075 and -0.15 with $S t_{1}=0.660$ and $S t_{2}=1.485$, corresponding to the first peak and second peak, respectively. However, at $X / D=-0.225$ the second peak disappears whereas the first peak amounts to $S t_{1}=0.660$ - this resembles the nondimensional frequency at the other two locations. In contrast to the pressure spectra, the spectrum derived from velocity components yield two peaks both in the free- and forced-vortex regions. The tangential velocity spectra exhibit two distinct peaks at all locations with lower harmonic corresponding to $S t_{1}=0.660,0.658$ and 0.663 , and the higher harmonic at $S t_{2}=1.485,1.470$ and 1.495 , respectively. The axial velocity pulsation spectra, as indicated in the third row of Fig. 7, indicates the first peak corresponding 
to $S t_{1}=0.661,0.663$ and 0.630 , and the second peak at $S t_{2}=1.512,1.469$ and 1.464 , respectively. As compared to the lower harmonic values, the difference is slightly larger in the higher harmonics. The last row in Fig. 7 presents the spectrum resolution based on radial velocity signals - the lower harmonic at the three locations is $S t_{1}=0.663$, whereas, the higher harmonic corresponds to $S t_{2}=1.495,1.443$, and 1.443 , respectively. The spectra derived from axial velocity and radial velocity signals exhibit multiple peaks at $X / D=-0.225$, possibly due to the increased influence of stochastic turbulence. Furthermore, the power of the spectrum reduces when moving radially outward, except for the tangential velocity pulsation spectra. This is due to the significant effect of PVC on fluctuating tangential velocity close to the walls (with limited contribution to the signal variance) as reported by Hoekstra (2000).

A similar procedure has been applied on the swirl tube to extract the spectra at same (normalized) radial locations (cf. Fig. 8). In contrast to the two spectral peaks in Stairmand cyclone, a single peak with $S t=0.511$ is extracted from the pulsation data of pressure and velocity components. In the region of turbulent fluctuations, the spectra derived from static pressure and axial velocity, yield multiple peaks. Such deviations in the static pressure and axial velocity spectrum were also noticed at $Z / D=-0.225$ in Stairmand cyclone.

as mentioned earlier, few studies had conjectured the first peak to evolve from the selfrotational effects of vortex core (Gu et al., 2016; Jia et al., 2019). However, this would require the inner vortex to possess periodicity about its rotational axis. Therefore, the instantaneous static pressure values are recorded at the vortex core centre over a plane $(Z / D=0.0)$ inside the vortex finder in Stairmand cyclone for a duration of $51.2 \mathrm{D} / U_{i n}$. The FFT is then performed over the resulting temporal signals for the periodicity check and presented in Fig. 9. The spectra demonstrate a nonperiodic behaviour of the vortex core about its centre of rotation. In another study, Gao et al. (2010) had mentioned the first peak as the frequency of outer vortex. To this, it is important to note that the power of the first spectral peak in Stairmand cyclone is the 
maximum in the core region and it diminishes significantly in the outer vortex - this is a clear indication that the lower harmonic does not relate to the outer vortex. Furthermore, $80 \%$ of the fluctuating kinetic energy in the core region is attributed to the coherent fluctuations (Hoekstra, 2000), and so the effect of turbulent fluctuations on the spectrum derived in the core region is minor.

To summarize, we considered monitoring temporal signals of different flow variables in both the free- as well as forced-vortex regions, and their spectral estimates were found to agree well with the experimental data. We have also discussed the nonperiodic behaviour of the vortex core centre (cf. Fig. 9) that showed no relation with any of the frequency peaks in the spectrum. Furthermore, the discussion on the minor influence of the turbulent fluctuations on the (high energy carrying) coherent structure confirms no dependency of the outer vortex motion on the spectral peaks derived in the core region. This is a clear indication that the frequency peak(s) in the spectrum do not relate to the (rotational) effects of either inner or outer vortex. Also, despite a similar flow physics, two spectral peaks in Stairmand cyclone and one peak in the swirl tube were extracted both in the experimental work (Hoekstra, 2000) and the present study. Had the motion of inner and/or outer vortex be responsible for the spectral peaks, the spectrum derived from the swirl tube would have also yielded two peaks as with the Stairmand cyclone model. It is interesting to notice the findings by Gronald and Derksen (2011) in which a single distinct peak with $S t=2.3$ was reported both in the experimental and simulation work.

Based on the above discussions, it appears that the complex precessing motion of the vortex core centre manifests itself as periodic signals and appear as two distinct spectral peaks in the frequency domain in Stairmand cyclone and a single peak in swirl tube. Therefore, the two distinct peaks in case of Stairmand cyclone and single peak in swirl tube are more likely due to the complex motion of the vortex core centre about the geometrical axis. 


\subsection{The dependency of spectral peaks on Re}

Fig. 10 represents the variations in the spectral peaks with $R e$ in Stairmand cyclone model. At $R e=0.87 \cdot 10^{5}, 1.74 \cdot 10^{5}, 2.80 \cdot 10^{5}, 3.48 \cdot 10^{5}$, and $4.35 \cdot 10^{5}$, the lower harmonic $\left(S t_{1}\right)$ corresponds to $0.639,0.636,0.670,0.660$, and 0.666 , whereas the higher harmonic $\left(S t_{2}\right)$ corresponds to $1.334,1.449,1.495,1.504$, and 1.512, respectively (cf. Fig. 10(a)). St 2 slightly increases with $R e$, whereas $S t_{1}$ is nearly a constant. On a magnified scale (cf. Fig. 10(b)), the behaviour of $S t_{2}$ with $R e$ reveals interesting facts. At first, the principal frequencies vary significantly with $R e$ ranging from $0.87 \cdot 10^{5}$ to $2.80 \cdot 10^{5}$. With further increase in $R e$ from $2.80 \cdot 10^{5}$ to $4.35 \cdot 10^{5}$, only a mild increase is noticed, and the curve tends to be asymptotic. Therefore, at higher $R e$, the precessional frequency of the inner vortex almost reaches a saturation level and gets Reynolds independent.

\subsection{The mean and RMS values at different Re}

In this section, the effect of $R e$ on the mean as well as RMS profiles of static pressure and velocity components are presented for Stairmand cyclone at $Z / D=0.60$ - this axial location is close to the lower lip of vortex finder. Static pressure takes up the maximum value near the wall and decreases nonlinearly when moving radially inwards. From Fig. 11(a) it becomes apparent that the radial pressure gradients confined to the inner vortex are significantly larger than in the outer vortex; therefore, the static pressure drops down rapidly in this zone. Since atmospheric conditions are imposed at the outlet boundary, a negative pressure exists at the rotational axis (determined from the zero-slope condition) of vortex core due to the strong swirl intensity. With the increase in $R e$ from $0.87 \cdot 10^{5}-2.80 \cdot 10^{5}$, a mild difference in the level of dip is noticed in the central region, whereas near the wall the pressure increases significantly. However, beyond $R e=2.80 \cdot 10^{5}$ the wall static pressure gets $R e$ independent. In the precession 
induced vortex motion, the intensity of pressure fluctuations in the free- and forced-vortex differ significantly. The pressure fluctuations reach to a maximum in the core region (due to direct influence of PVC), whereas in the outer vortex region mild fluctuations are noticed. Steeper slopes in RMS static pressure profile are observed in the inner vortex region (cf. Fig. 11(b)) whereas these are nearly a constant in the outer vortex - the slopes change noticeably in the region connecting the outer vortex and inner vortex. In the outer vortex region, the effects of coherent pressure fluctuations are much weaker and dominated mainly by the turbulent fluctuations. Over a given range of $R e$, the RMS static pressure profile nearly overlaps and does not exhibit large differences - this is in contrast to what was observed in the mean pressure values.

Figs. 11(c) and 11(d) illustrates the effect of $R e$ on the mean and RMS values of tangential velocity, respectively. Two regions, based on the mean velocity profiles, are distinguishable: the forced vortex region where the tangential velocity varies linearly and a free vortex region where the velocity decays when moving out in the radial direction. In the outer region of vortex core - determined from the zero slope at the points of maxima and minima in the mean tangential velocity (cf. Fig. 11) - an increase in the peak value of tangential velocity magnitude is observed at $R e$ ranging from $0.87 \cdot 10^{5}-2.80 \cdot 10^{5}$. With further increase in $R e$, no significant changes are noticed. Due to the influence of PVC, the tangential velocity fluctuations are strong in the core region than in the outer vortex region. Over the entire range of $R e$, the RMS tangential velocity profiles change slightly in the stochastic turbulence region.

The mean and RMS profiles of axial velocity are elucidated in Figs. 11(e) and 11(f), respectively. The mean axial velocity resembles the shape of an inverted $\mathrm{W}$ profile. The negative values indicate flow in the downward direction, whereas the positive value indicates an upwardly directed flow. A dip in the profile near the geometrical axis is due to adverse pressure gradients in the core region. For all $R e$, the mean axial velocity profiles almost overlap 
and exhibits small changes in the near-wall region. As with the static pressure and tangential velocity, the level of axial velocity fluctuations is highest in the core region than in the outer vortex. In contrast to the behaviour of mean axial velocity, the RMS profiles are largely affected by $R e$ and undergo appreciable changes both in the free- and forced-vortex region.

The behavioural pattern of the higher harmonic (St2) with Re (cf. Fig. 10(b)) could now be explained by relating it with the pressure and velocity profiles. Over a range of $R e$ from $0.87 \cdot 10^{5}-2.80 \cdot 10^{5}$, significant changes in $S t_{2}$ are observed - this agrees well to what has been observed in the (nature of) mean profiles of static pressure and tangential velocity components. Therefore, a correlation exists between PVC and the mean profiles of pressure and velocity.

\subsection{The mean and RMS values along the geometrical axis}

At the rotational axis of the vortex core, the mean static pressure and tangential velocity possess the lowest values, whereas the axial velocity exhibits a local dip (cf. Fig. 11). Since the axis of vortex core takes a spiral shape around the geometrical axis (cf. Fig. 6(f)), the mean and RMS values of pressure and velocity components - depending on the slope of their respective profiles and offset between the two axes - are likely to change.

Figs. 12(a) and 12(b) present variations in mean and RMS static pressure values along the centreline. At the outlet plane the fluid escapes into the atmosphere at zero (gauge) pressure (cf. Fig. 12(a)) and reduces significantly till half the protruding length of the outlet tube. Inside the vortex finder and collection bin (including the region of cone tip), the differences in mean pressure values are large. On the other hand, the pressure fluctuations at the outlet plane are negligible due to atmospheric conditions at the outlet plane - thereafter it increases rapidly at a small distance from this plane inside the vortex finder tube. As in the outlet tube, pressure fluctuations are high inside the bin. However, in the separation space, these values do not differ much. 
Figs. 12(c-f) illustrate the mean and RMS values of tangential and axial velocities along the centreline. The variations in the mean tangential velocity are stronger than mean axial velocity, whereas their maximum RMS values are comparable. The two velocity components undergo a large change in the separation space (in contrast to the observations for the mean pressure). The mean tangential velocity varies significantly inside the bin due to greater offset of the vortex near the bottom plate. The RMS value of tangential velocity in the bin is comparable to that in the separation region of the cyclone. The mean axial velocity values largely vary inside the vortex finder tube - especially downstream to the lower lip of the tube. This is due to a sudden contraction in the main flow that increases the axial velocity to satisfy the continuity equation. Much lower fluctuation levels are noticed inside the bin and near the bottom plate. In the separation space, the mean values of tangential velocity and axial velocity change significantly throughout the cyclone length.

It becomes apparent from Fig. 12 that a small region both upstream and downstream to the cone tip experiences larger changes in pressure and velocity values. In this region, the flow in outer vortex expands inside the bin and the flow from the bin contracts as it moves up in the core region. Due to this - and more due to single inlet - the vortex core gets asymmetric near the cone tip and inside the collection bin that exhibits large changes in both mean and fluctuating pressure and velocity values.

\section{Conclusions}

The present study makes use of numerical simulations on the single-phase fluid flow in two different types of reverse-flow cyclone separators viz. Stairmand cyclone with $D=0.29 \mathrm{~m}$ and a swirl tube with $D=0.1 \mathrm{~m}$ to examine the complex flow behaviour. Large-eddy simulation (LES) was performed on grids fine enough to resolve the flow features that appear in experiments, especially the vortex core precession - a phenomenon that is often observed in swirl- 
dominating flows. With Stairmand cyclone, a vast experimental data sets are available; therefore, the geometry resembling this cyclone model was analysed for validation of the simulation methodology. The LES predictions for both mean as well as RMS values of the tangential and axial velocity components agreed well with the experimental data. Furthermore, two distinct spectral peaks predicted by the numerical simulations closely resembled the experimental values. At $R e=2.8 \cdot 105$, the numerically simulated Strouhal number for the (first) low-frequency peak $\left(S t_{1}\right)$ was 0.670 against the experimental value of 0.7 , whereas the second frequency peak $\left(S t_{2}\right)$ was 1.495 against the experimental value of 1.58 , which differs by nearly $5 \%$.

First, the general behaviour of the vortex core was discussed. By analysing the realizations of static pressure contour plots, it becomes apparent that the vortex centre precesses at a small average distance from the geometrical axis. Furthermore, the path traced out by the least (instantaneous) static pressure values - recorded on a plane $Z / D=0.0$ - demonstrated a complex path of the vortex core centre. Based on this data, the probability density function (PDF) plot was extracted that indicated the spatial transition (from the geometrical axis) of the vortex centre both in $\mathrm{x}$ - as well as $\mathrm{y}$-directions. It was further shown that the vortex centre has a preferred angular location close to $1.5 \pi$ radians. Throughout the cyclone length, an offset of the inner vortex was observed.

The precessing frequency of vortex core was estimated at different radial locations viz. $X / R=-0.15,-0.30$ and -0.45 , all at $Z / D=0.0$ and $Y / D=0.0$, inside Stairmand cyclone - these radial locations covered both the free- and forced-vortex regions. FFT was performed on the temporal signals of static pressure, tangential velocity, axial velocity and radial velocity at $R e=2.8 \cdot 10^{5}$ the dual peaks in the spectra showed close resemblance with the experimental values. At all the radial locations, the respective estimated nondimensional frequency values of $S t_{1}$ as well as $S t_{2}$ - derived from all the signals - were nearly the same. A similar study was conducted on the 
swirl tube at $R e=1.4 \cdot 10^{4}$ - a single distinct peak of $S t_{P V C}=0.511$ was noticed in the spectrum derived from all temporal signals that agreed well with the experimental value of $S t=0.49$. Here, we have two important observations. First, despite a similar flow physics, the Stairmand cyclone showed two distinct peaks, whereas the swirl tube revealed a single spectral peak. Second, the same frequency values - irrespective of the free and forced vortex zones - exist for all temporal signals. This indicates no dependency of the periodicity (associated with the flow feature) on the two zones.

In addition to the above, the periodicity of the inner vortex about its rotational axis is also evaluated. For this, the time-dependent signals of minimum static pressure were captured over the plane $Z / D=0.0$ for spectral estimates. The FFT of the captured signal revealed a nonperiodic behaviour of the vortex core centre. It is interesting to notice the nonperiodic behaviour of the inner vortex about the rotational axis and periodic behaviour due to its precessional motion about the geometrical centre. This indicates that the spectral peaks are not related to the rotation of the inner and outer vortex regions (as revealed by several earlier studies); rather, the complex precessing motion of the vortex core centre manifests itself as periodic signals that appear as two distinct spectral peaks in the frequency domain in Stairmand cyclone and a single peak in swirl tube.

The dependency of the two spectral peaks inside Stairmand cyclone on $R e$ (in the range $0.87 \cdot 10^{5}$ to $4.35 \cdot 10^{5}$ ) was examined. It was observed that $S t_{2}$ marginally increases with $R e$, whereas the variation of $S t_{1}$ is nearly a constant. A detailed analysis revealed that at higher $R e$ the second frequency mode $\left(S t_{2}\right)$ gets Reynolds independent. A similar effect of $R e$ on the mean profiles of static pressure and tangential velocity was observed - this confirms the correlation between the PVC and mean profiles. Lastly, the variations in mean and RMS values of pressure and velocity components were evaluated along the geometrical axis. The large differences were 
observed due to change in the slopes of both mean and RMS values of the flow variables at the geometrical axis.

\section{Acknowledgement}

The authors wish to thank A.J. Hoekstra (T.U. Delft) for making available the experimental data.

\section{References}

Akiyama, O., and Kato, C., 2017, "Numerical Investigations of Unsteady Flows and Particle Behavior in a Cyclone Separator," ASME J Fluid Eng, 139(9), pp. 091302-091313. (https://doi.org/10.1115/1.4036589).

Alekseenko, S.V., Kuibin, P.A., and Okulov, V.L., 2007, Theory of Concentrated Vortices: An Introduction, Springer Science \& Business Media. (DOI: 10.1007/978-3-540-73376-8)

Brar, L.S., and Elsayed, K., 2017, "Analysis and optimization of multi-inlet gas cyclones using large eddy simulation and artificial neural network," Powder Tech, 311, pp. 465-483. (https://doi.org/10.1016/j.powtec.2017.02.004). 
Brar, L.S., and Elsayed, K., 2018, "Analysis and optimization of cyclone separators with eccentric vortex finders using large eddy simulation and artificial neural network," Sep Puri Tech, 207, pp. 269-283. (https://doi.org/10.1016/j.seppur.2018.06.013).

Chorin, A.J., 1968, "Numerical solution of the Navier-Stokes equations," Math Comp, 22(104), pp. 745-762. (https://doi.org/10.1090/S0025-5718-1968-0242392-2).

de Souza, F.J., de Vasconcelos Salvo, R., and de Moro Martins, D.A., 2012, "Large Eddy Simulation of the gas-particle flow in cyclone separators," Sep Puri Tech, 94, pp. 61-70. (https://doi.org/10.1016/j.seppur.2012.04.006).

Derksen, J.J., 2003, "Separation performance predictions of a Stairmand high-efficiency cyclone," AIChE J, 49(6), pp. 1359-1371. (https://doi.org/10.1002/aic.690490603).

Derksen, J.J., and Van den Akker, H.E.A., 2000, "Simulation of vortex core precession in a reverse-flow cyclone," AIChE J, 46, pp. 1317-1331. (https://doi.org/10.1002/aic.690460706).

Dhakal, T.P., Walters, D.K., and Strasser, W., 2014, "Numerical study of gas-cyclone airflow: an investigation of turbulence modelling approaches," Int J Comp Fluid Dyn, 28(1-2), pp. 1-15. (https://doi.org/10.1080/10618562.2013.878800).

Elsayed, K., and Lacor, C., 2011, "The effect of cyclone inlet dimensions on the flow pattern and performance," App Math Model, 35(4), pp. 1952-1968.

(https://doi.org/10.1016/j.apm.2010.11.007). 
Ferziger, J.H., and Perić, M., 2002, Computational methods for fluid dynamics, Berlin, Springer. (https://doi.org/10.1007/978-3-642-56026-2).

Froud, D., O'Doherty, T., and Syred, N., 1995, "Phase averaging of the precessing vortex core in a swirl burner under piloted and premixed combustion conditions," Comb Flame, 100(3), pp. 407-412. (https://doi.org/10.1016/0010-2180(94)00167-Q).

Gao, C., Sun, G., Dong, R., and Fu, S., 2010, "Characterizing the dynamic property of the vortex tail in a gas cyclone by wall pressure measurements," Fuel Proc Tech, 91(8), pp. 921926.

(https://doi.org/10.1016/j.fuproc.2009.12.019).

Gao, Z., Wang, J., Wang, J., and Mao, Y., 2019, "Time-frequency analysis of the vortex motion in a cylindrical cyclone separator," Chem Eng J, 373, pp. 1120-1131. (https://doi.org/10.1016/j.cej.2019.05.054).

Gronald, G., and Derksen, J.J., 2011, "Simulating turbulent swirling flow in a gas cyclone: A comparison of various modeling approaches," Powder Tech, 205(1-3), pp. 160-171.

(https://doi.org/10.1016/j.powtec.2010.09.007).

Gu, X., Song, J., and Wei, Y., 2016, "Experimental study of pressure fluctuation in a gas-solid cyclone separator," Powder Tech, 299, pp. 217-225.

(https://doi.org/10.1016/j.powtec.2016.05.028).

Hinze, J.O., 1975, Turbulence, McGraw-Hill Publishing Co., NY. 
(https://doi.org/10.1017/S0022112077211864).

Hoekstra, A.J., 2000, "Gas flow field and collection efficiency of cyclone separators," Ph.D. dissertation, Delft University of Technology, Netherlands.

(http://resolver.tudelft.nl/uuid:67b8f405-eef0-4c2d-9646-80200e5274c6).

Hoekstra, A.J., Israël, A.T., Derksen, J.J., and Van den Akker, H.E.A., 1998, "The application of laser diagnostics to cyclonic flow with vortex precession," In Int Symp Applications of Laser Techniques to Fluid Mechanics, Lisbon.

Jia, M., Wang, D., Yan, C., Song, J., Han, Q., Chen, F., and Wei, Y., 2019, "Analysis of the pressure fluctuation in the flow field of a large-scale cyclone separator," Powder Tech, 343, pp. 49-57. (https://doi.org/10.1016/j.powtec.2018.11.007).

Levy, Y., and Albagli, 1991, "Experimental study on confined two-phase jets," J thermal heat trans, 5(3), pp. 387-393. (https://doi.org/10.2514/3.275).

Liu, Z., Emmanuel, H., Olsen, M.G., Hill, J.C., and Fox, R.O., 2017, "Turbulent mixing in the confined swirling flow of a multi-inlet vortex reactor," AIChE J, 63(6), pp. 2409-2419. (https://doi.org/10.1002/aic.15572).

Morse, O.M., 1886, “Dust Gullbgtor.” Patent DRP Nr. 39 219, 25.07.1886, Staubsammler, technical report, Knickerbocker Company, Jackson, USA.

Patankar, S., 2018, Numerical heat transfer and fluid flow, CRC press. 
Prandtl, L., 1925, "Bericht uber die entstehung der turbulenz," Zeitschrift f'ur Angewandte Mathematik und Mechanik, 5, pp. 136-139.

Shukla, S.K., Shukla, P., and Ghosh, P., 2013, "The effect of modeling of velocity fluctuations on prediction of collection efficiency of cyclone separators," Appl Math Model, 37(8), pp. 5774-5789. (https://doi.org/10.1016/j.apm.2012.11.019).

Smagorinsky, J., 1963, "General circulation experiments with the primitive equations: I. The basic experiment," Monthly weather review, 91(3), pp. 99-164. (https://doi.org/10.1175/15200493(1963)091\%3C0099:GCEWTP\%3E2.3.CO;2).

Solero, G., and Coghe, A., 2002, "Experimental fluid dynamic characterization of a cyclone chamber," Exp Therm Fluid Sci, 27(1), pp. 87-96. (https://doi.org/10.1016/S08941777(02)00221-2).

Sommerfeld, M., and Qiu, H.H., 1993, "Characterization of particle-laden, confined swirling flows by phase-doppler anemometry and numerical calculation," Int J Multi Flow, 19(6), pp. 1093-1127. (https://doi.org/10.1016/0301-9322(93)90080-E).

Stairmand, C.J., 1951, "The design and performance of cyclone separators," Trans Inst Chem Eng, 29, pp. 356-383. 
Temam, R., 1969, "Sur l'approximation de la solution des équations de Navier-Stokes par la méthode des pas fractionnaires (II)," Archive for Rational Mechanics and Analysis, 33(5), pp. 377-385. (https://doi.org/10.1007/BF00247696).

Wendt, J.F., 2008, Computational Fluid Dynamics: An Introduction, Springer Science \& Business Media. (DOI: 10.1007/978-3-540-85056-4).

Yazdabadi, P., Griffiths, A.J., and Syred, N., 1994, "Investigations into the precessing vortex core phenomenon in cyclone dust separators," Proc. Inst Mech Eng, Part E, J Proc Mech Eng, 208(2), pp. 147-154. (https://doi.org/10.1243\%2FPIME_PROC_1994_208_221_02). 

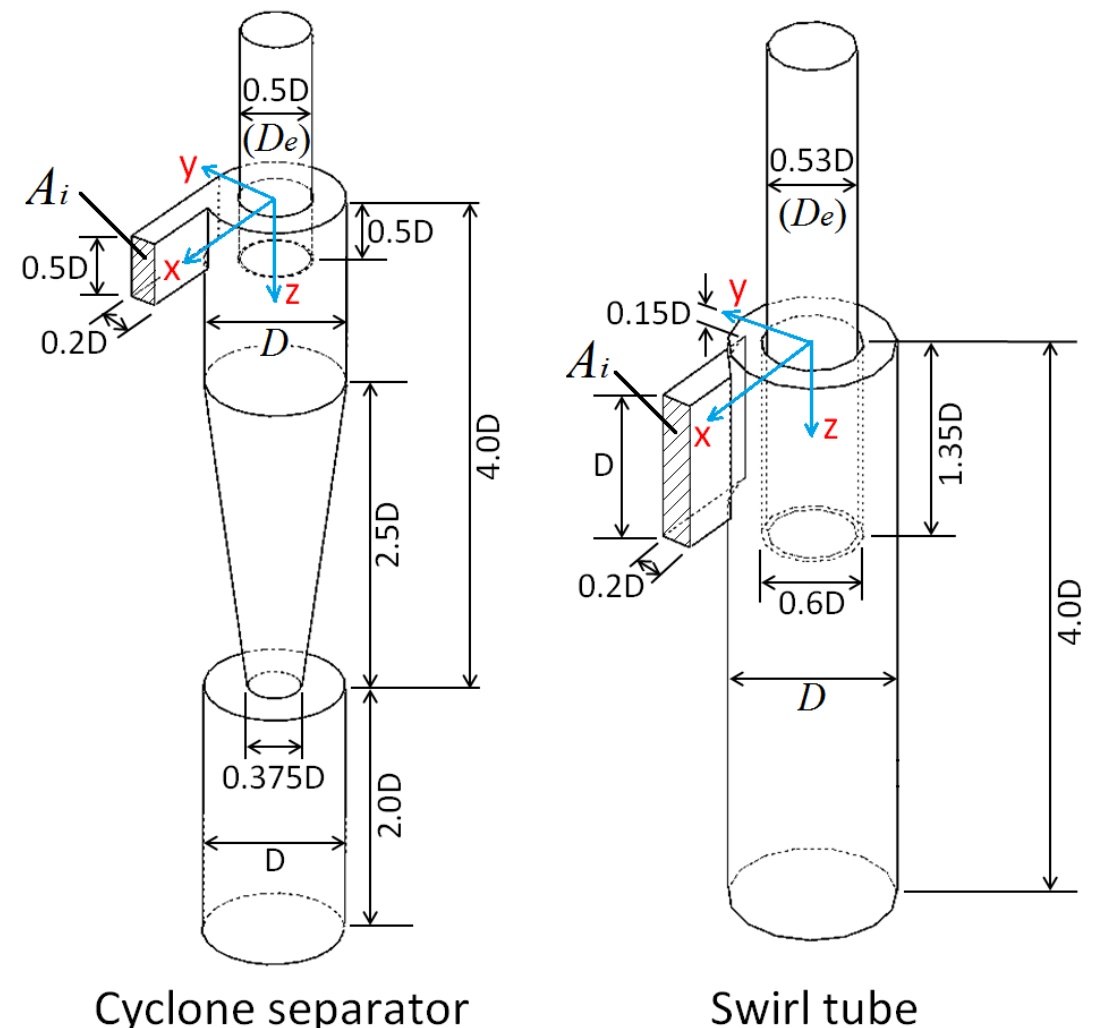

Swirl tube

Fig. 1 Geometrical details of Stairmand high-efficiency cyclone model (on the left) with $D=0.29 \mathrm{~m}$, and swirl tube (on the right) with $D=0.1 \mathrm{~m}$ 

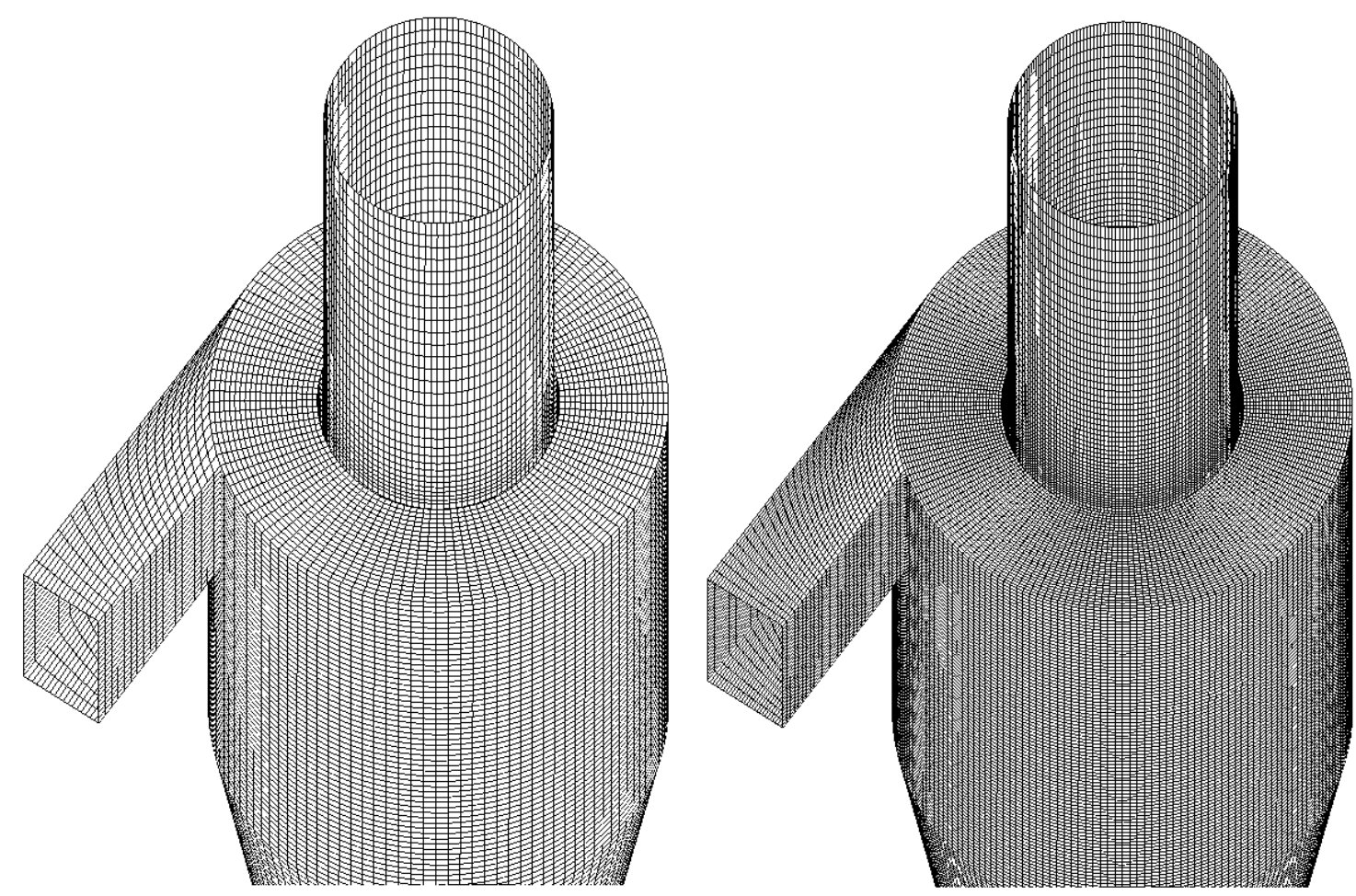

Fig. 2 Details of the mesh containing nearly 0.7 million hexahedra (to the left) and 2.9 million hexahedra (to the right) of Stairmand cyclone model $(D=0.29 \mathrm{~m})$. At the inlet section block, the number of nodes in the axial direction are 20 and 32, number of nodes in radial direction are 20 and 36, and number of nodes in tangential direction are 14 and 32, for coarse mesh (to the left) and fine mesh (to the right), respectively. 
Mean velocity components
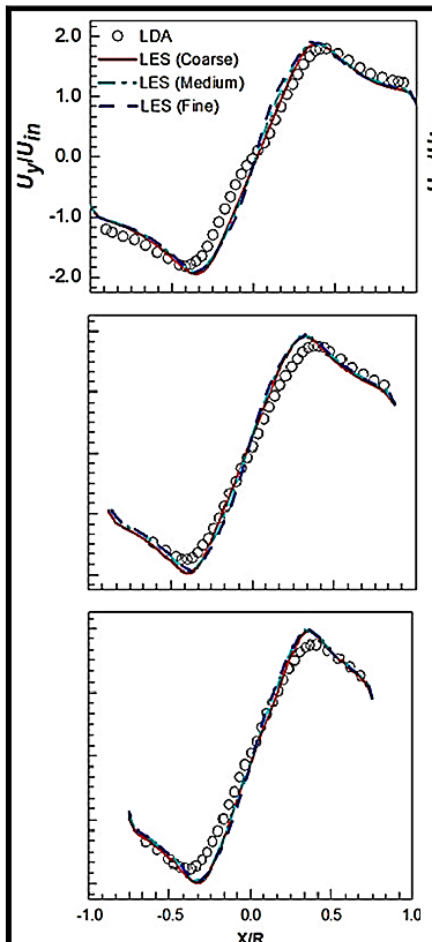
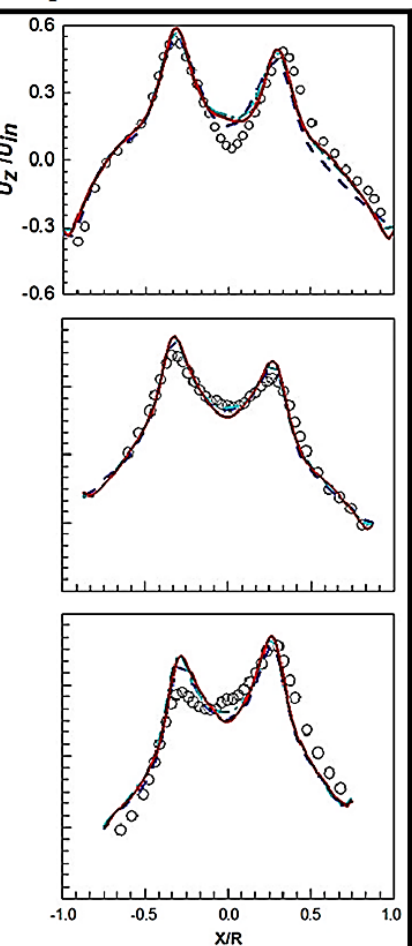

RMS velocity components
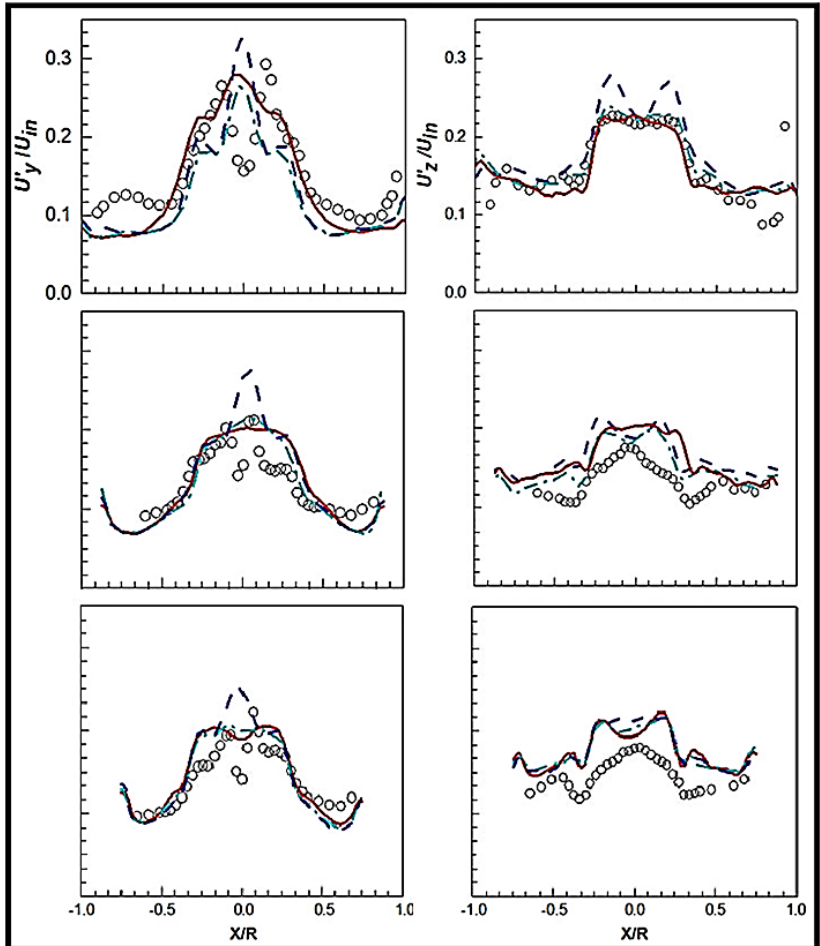

Fig. 3 The radial profiles measured along the lines $X / 2 R= \pm 1.0, Y / 2 R=0$ in Stairmand cyclone at $R e=2.8 \cdot 10^{5}$ for three levels of grid. From top to bottom: axial locations $Z / D=0.75,2.00$ and 2.50 . From left to right: mean $Y$ velocity and mean $\mathrm{Z}$ velocity (in the left block); RMS Y velocity, and RMS Z velocity (in the right block), respectively. The radial distance $X$ has been normalized with main body radius, $R=D / 2$. 
PSD plots at different levels of the grid

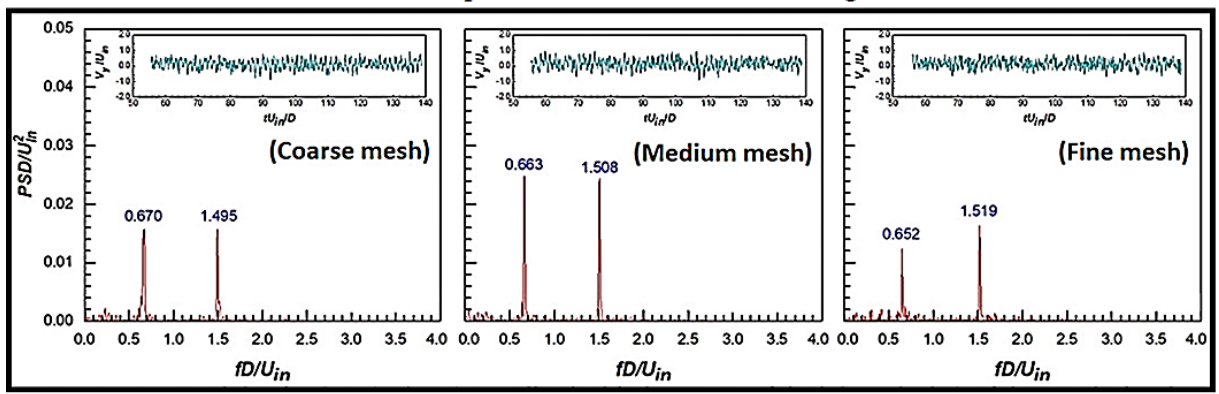

PSD plots at different time steps

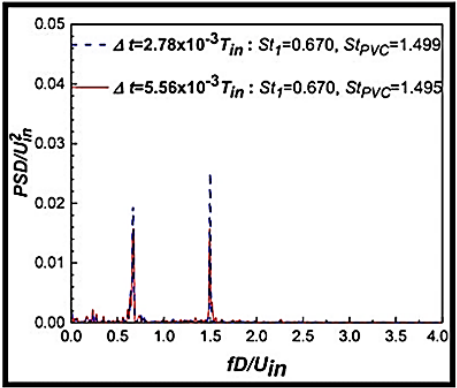

Fig. 4 Power spectral density (PSD) plots (normalized with the square of the inlet velocity) of the Y velocity-time series data recorded at $X / D=Y / D=Z / D=0$ at $R e=2.8 \cdot 10^{5}$ in Stairmand cyclone model. Left block: for three levels of grid viz. (a) 0.7 million hexahedra, (b) 1.8 million hexahedra and (c) 2.9 million hexahedra (in left block); and for two different time steps of $5.56 \cdot 10^{-3} T_{\text {in }}$ and $2.78 \cdot 10^{-3} T_{\text {in }}$ on the coarse mesh in a time frame $55.52 T_{\text {in }}-138.79 T_{\text {in }}$ (in right block), respectively. 

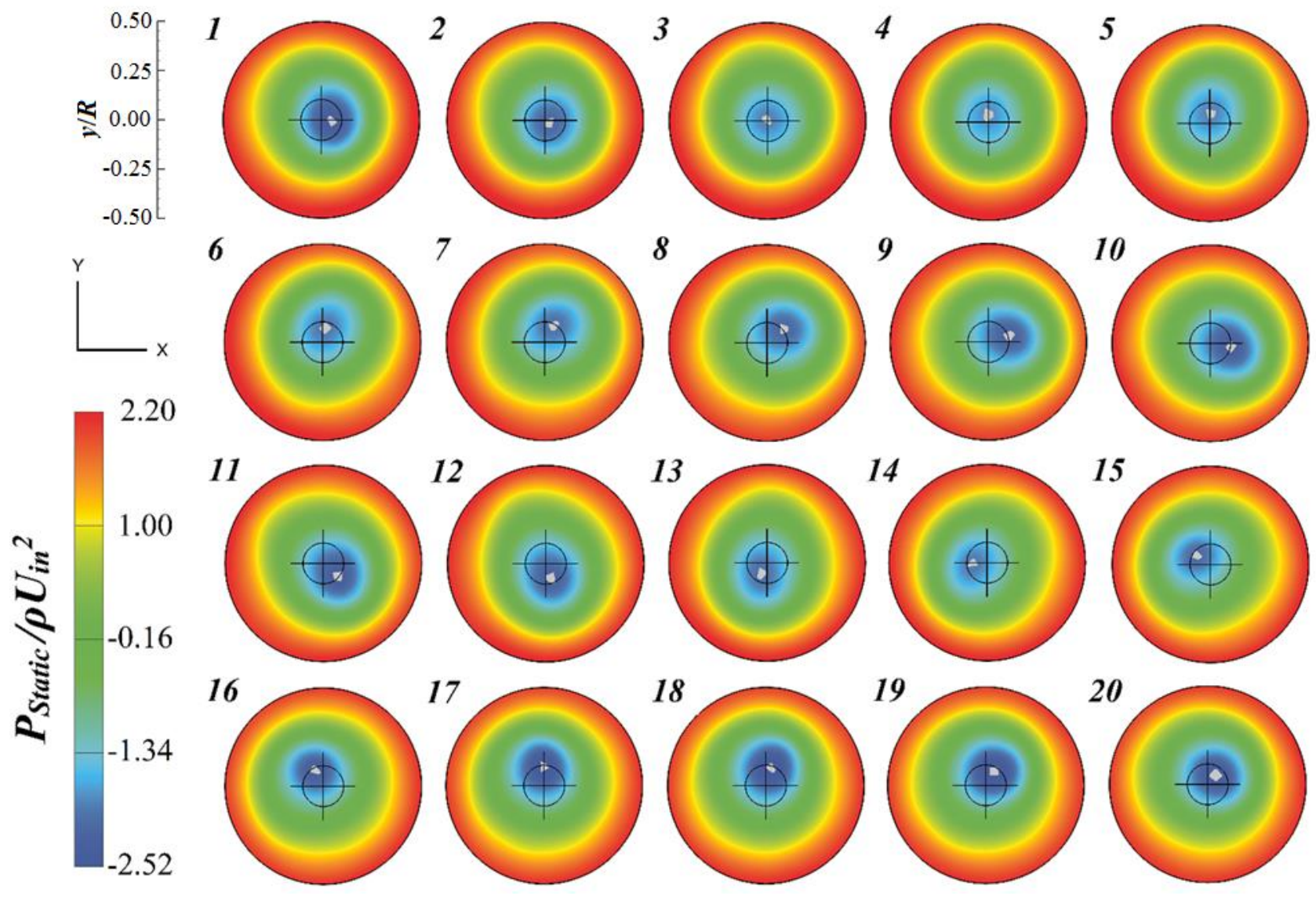

Fig. 5 Realizations of the static pressure field with time spacing of $0.067 D / U_{\text {in }}$ between subsequent images captured on a horizontal plane, $Z / D=0.0$ (inside the vortex finder). This time gap is nearly one-tenth of the duration taken by the vortex core centre to precess about the geometrical axis of cyclone. 

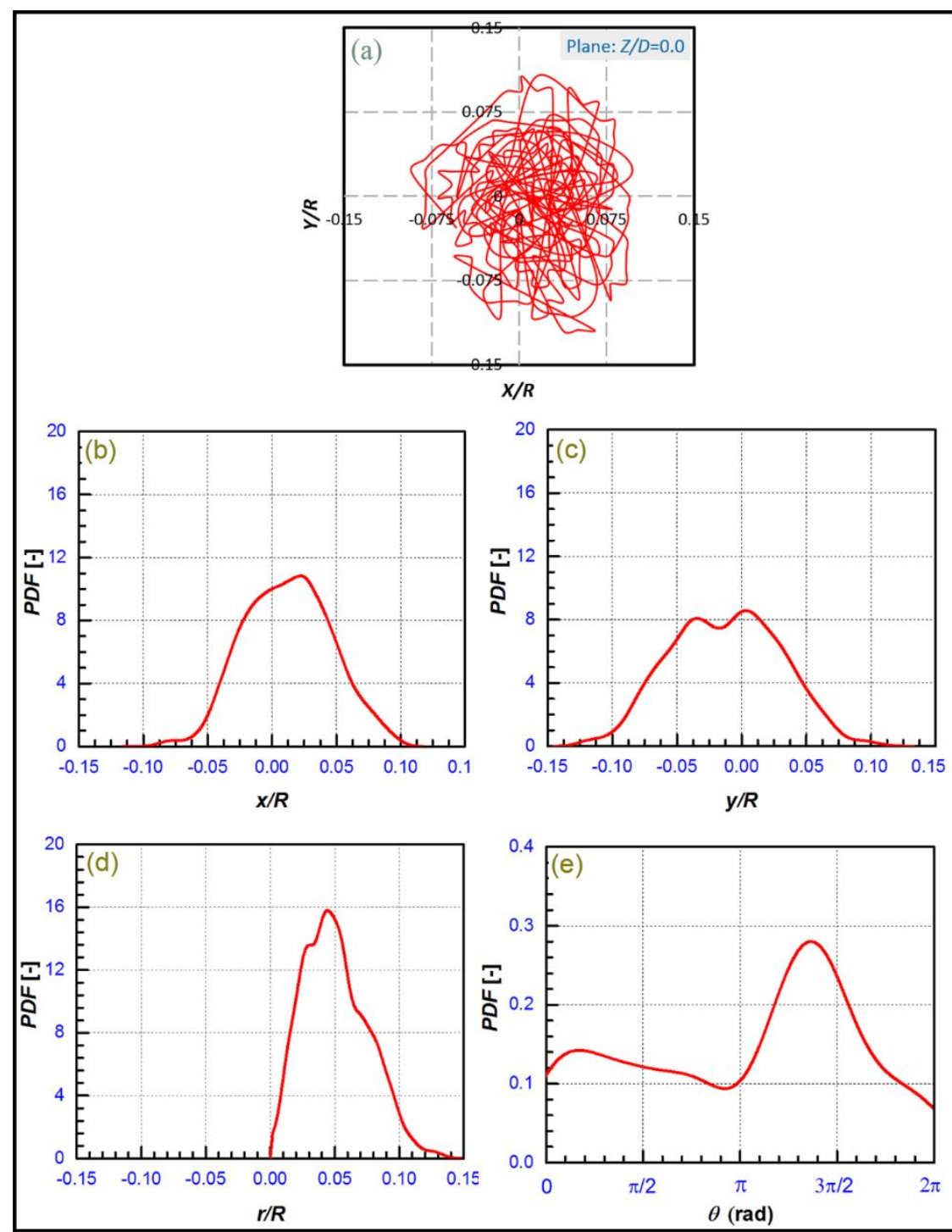

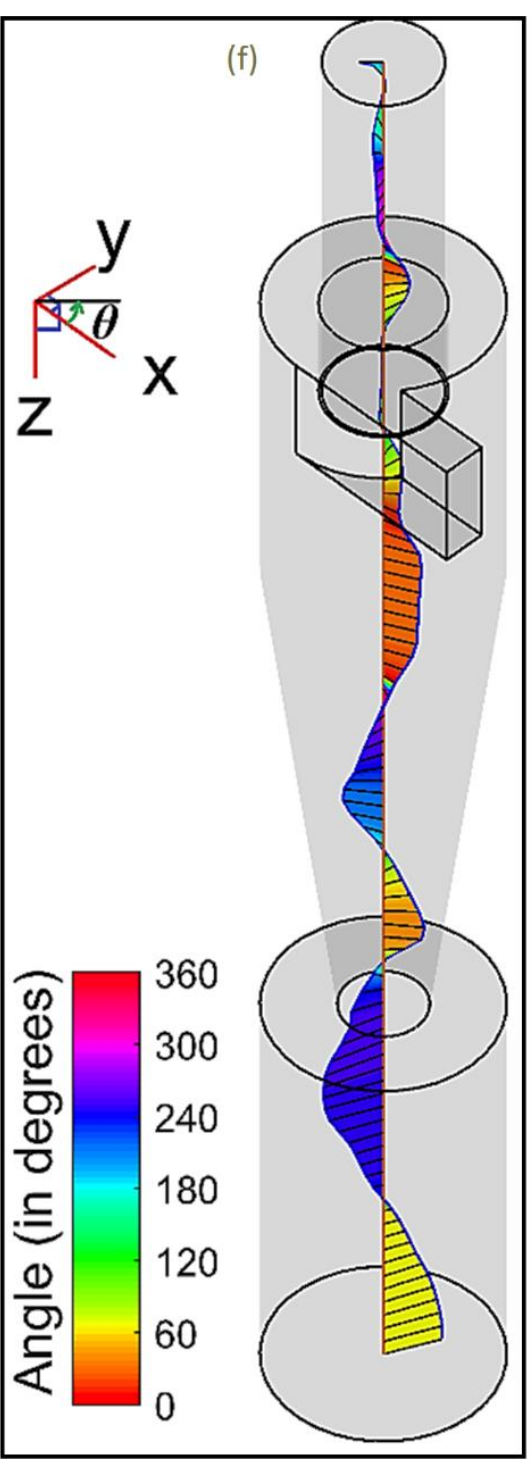

Fig. 6 Left block: The path followed by vortex core during the time frame of $17.21 \mathrm{D} / \mathrm{U}_{\text {in }}$ on a plane at $Z / D=0.0$; the traces correspond to the locus of points possessing least (instantaneous) static pressure recorded at each time step in Stairmand cyclone model. Probability density function of (a) $x$-position, (b) $y$-position, (c) $r$-position, and (d) angular position $(\theta)$ of the vortex core centre at the location $Z / D=0.0$.

Right block: The average distance of the vortex core centre from the geometrical axis of cyclone (Scale: $X \equiv 5: 1$, $\mathrm{Y} \equiv 5: 1$, and $\mathrm{Z} \equiv 1: 1)$. The colormap represents the angle of orientation with rotation taken positive in the counterclockwise direction from $X$-axis (that represents $0^{\circ}$ angle). 

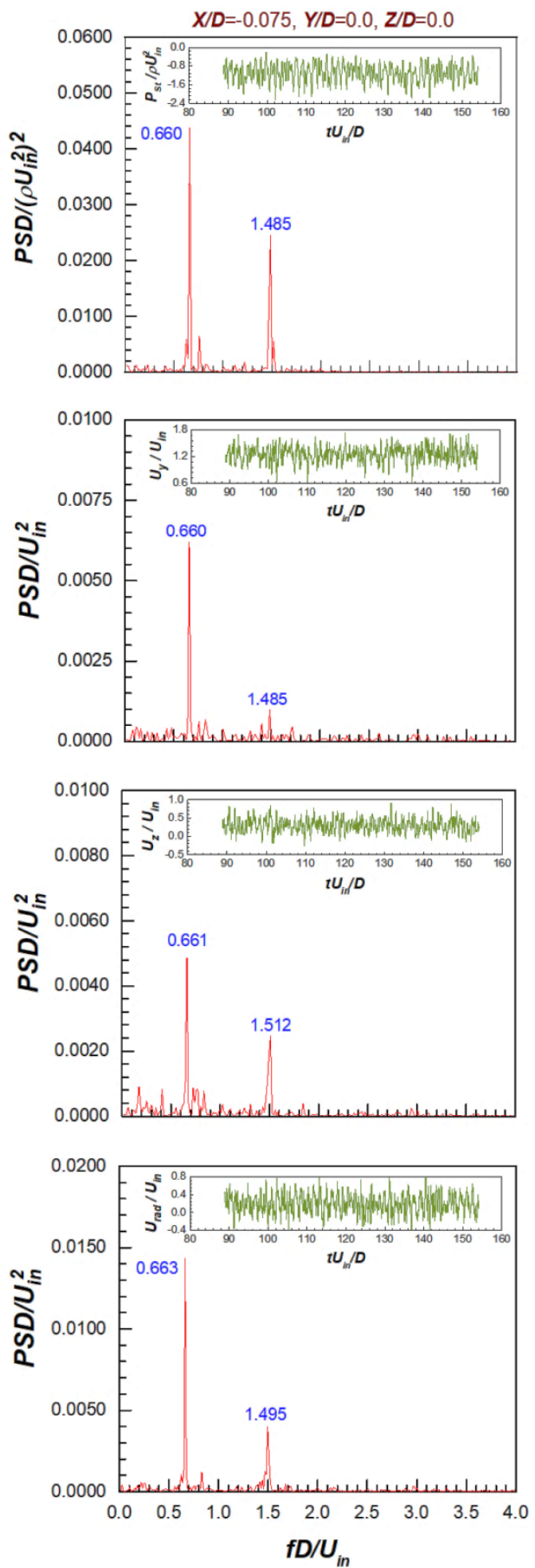
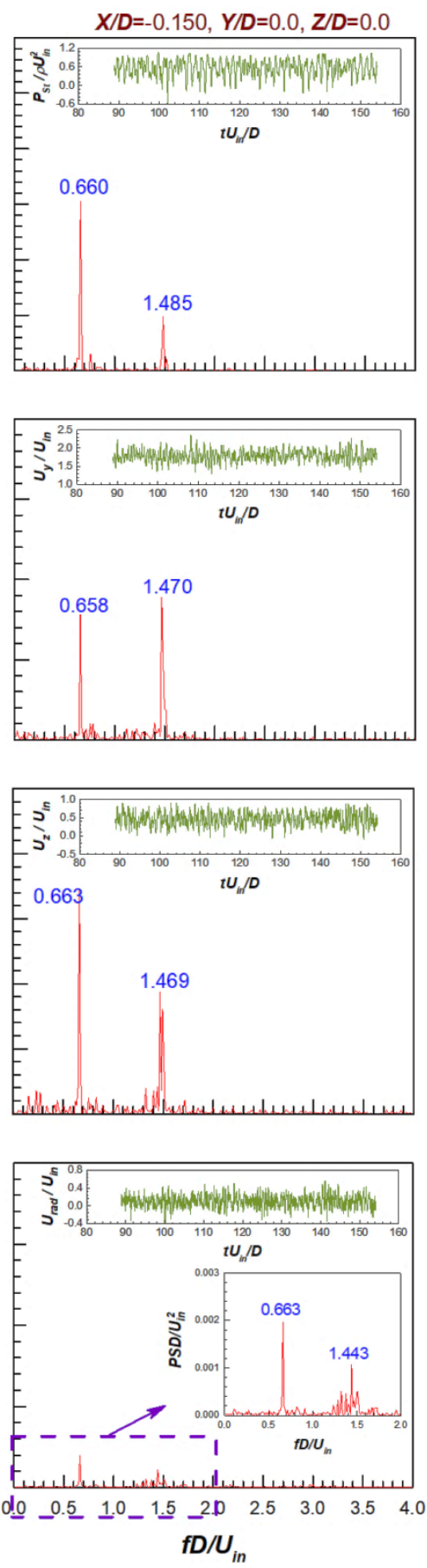
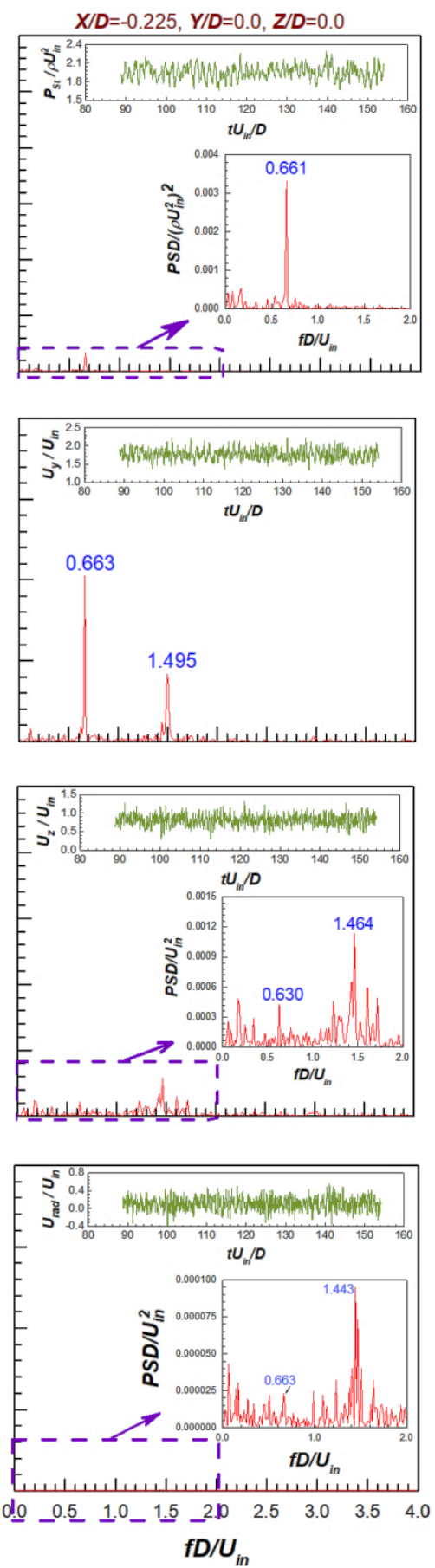

Fig. 7 PSD plots based on; from top to bottom: (a) static pressure-time series data, (b) tangential velocity-time series data, (c) axial velocity-time series data, and (d) radial velocity-time series data; from left to right: (a) at $X / D=-0.075$, (b) at $X / D=-0.150$, and (c) at $X / D=-0.225$; all signals recorded at $Y / D=0.0$, and $Z / D=0.0$. 

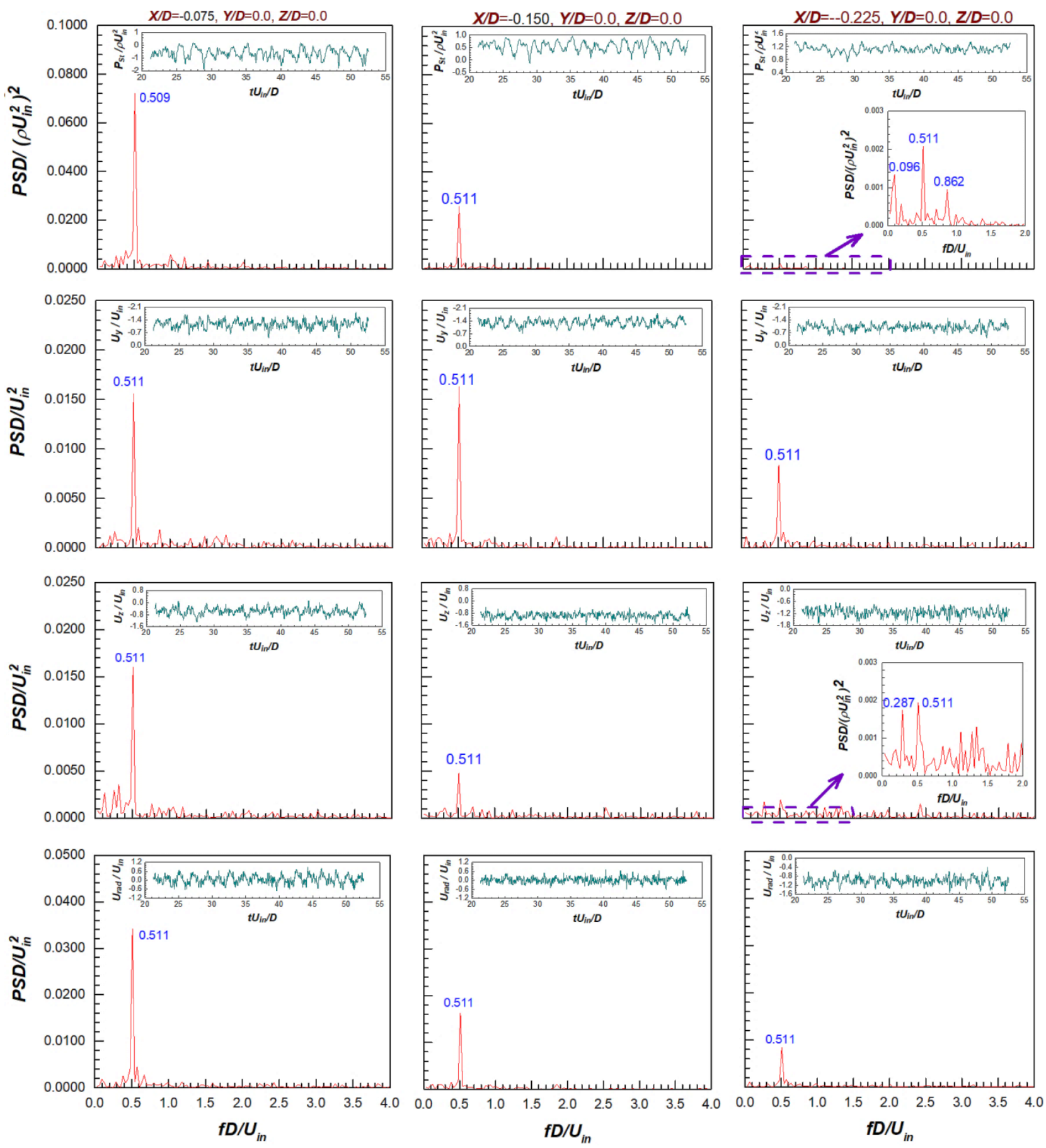

Fig. 8 PSD plots based on; from top to bottom: (a) static pressure-time series data, (b) tangential velocity-time series data, (c) axial velocity-time series data, and (d) radial velocity-time series data; from left to right: (a) at $X / D=-0.075$, (b) at $X / D=-0.150$, and (c) at $X / D=-0.225$; all signals recorded at $Y / D=0.0$, and $Z / D=0.0$. 


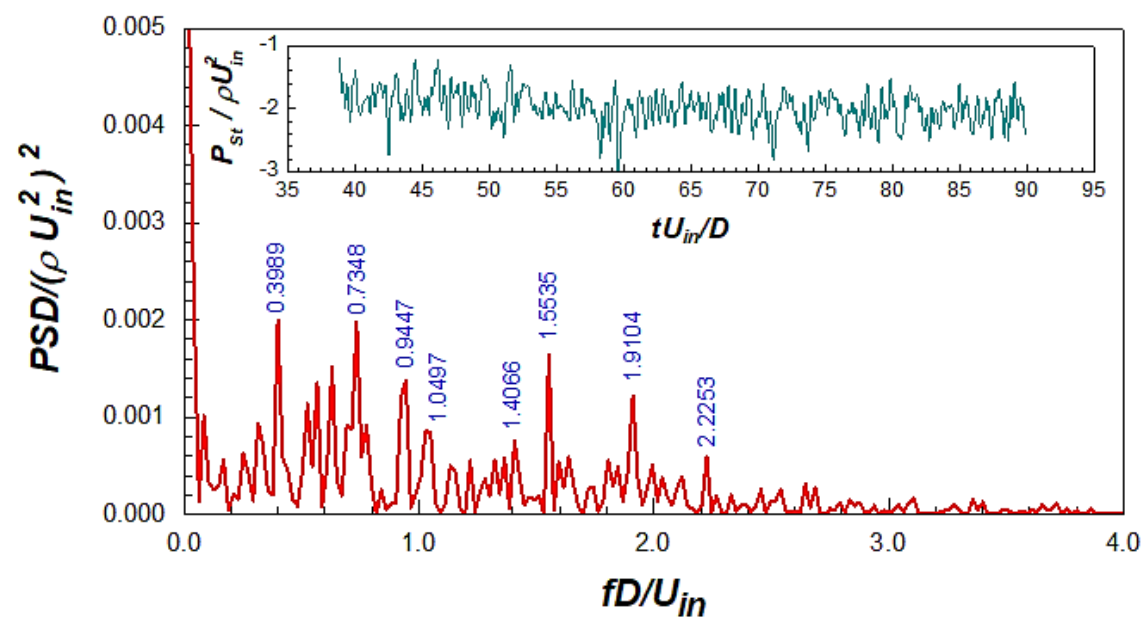

Fig. 9 FFT of the pressure signals recorded at the centre of the precessing vortex core in Stairmand cyclone at $Z / D=0.0$ for $R e=2.8 \cdot 10^{5}$. 

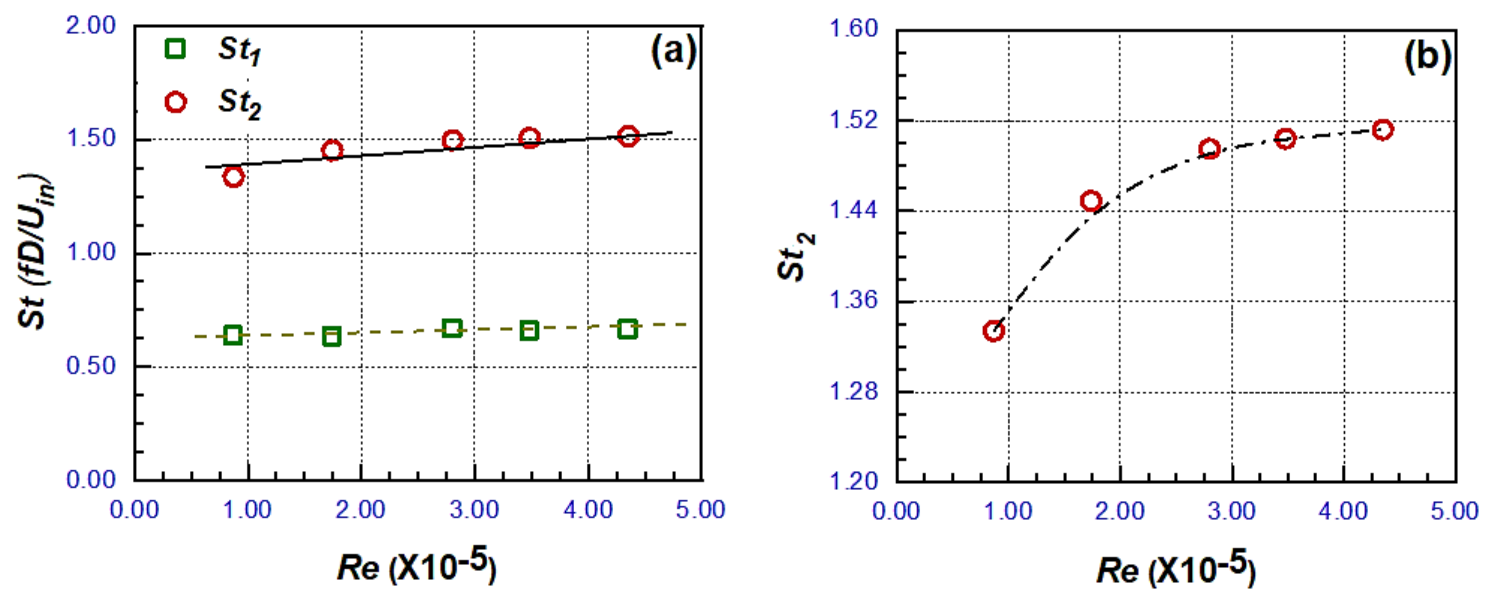

Fig. 10 From left to right: (a) variation of $S t_{1}$ and $S t_{2}$ with $R e$, and (b) variation of $S t_{2}$ with $R e$ on magnified scale in Stairmand cyclone model. 

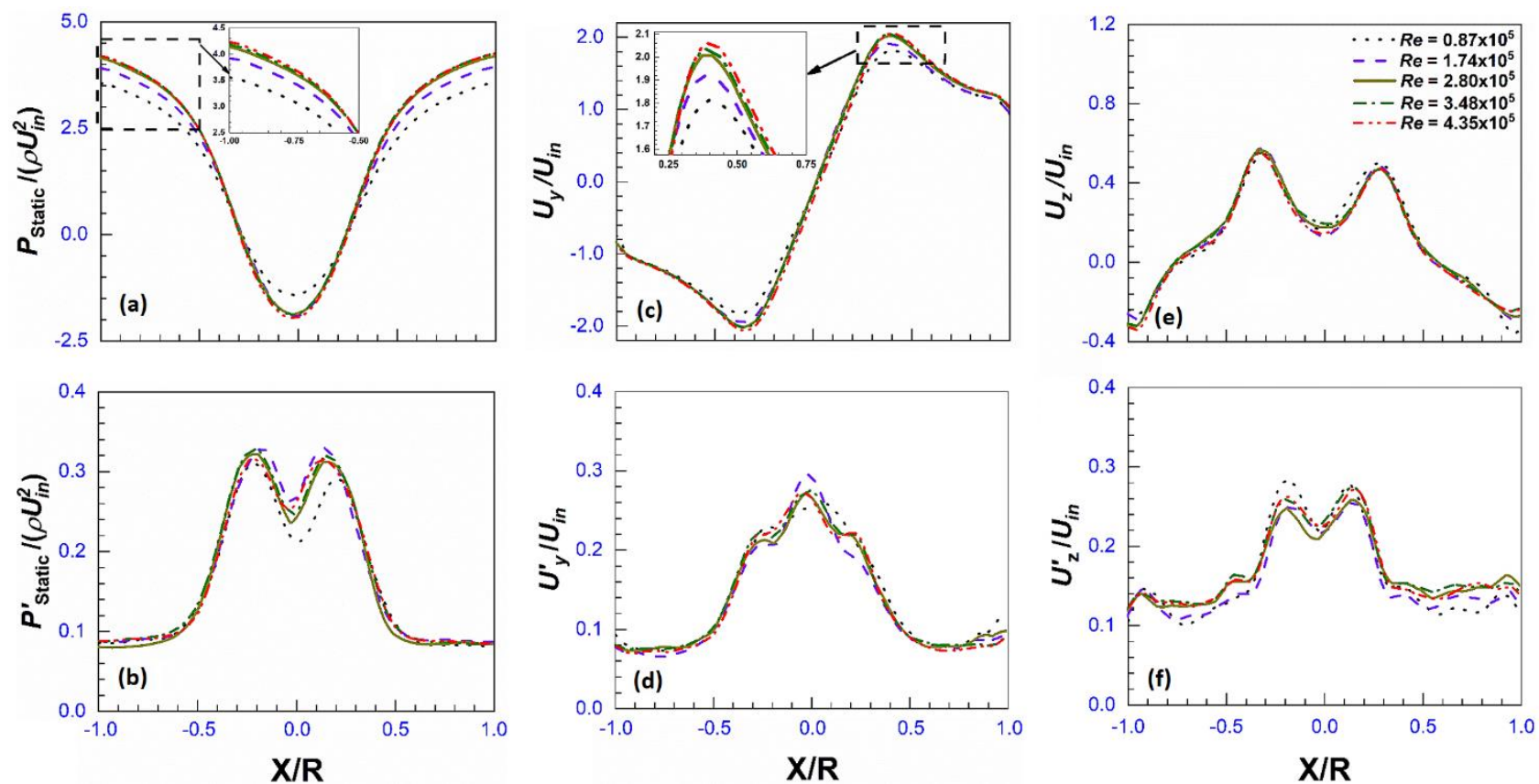

Fig. 11 Radial profiles at $X / D= \pm 1, Y / D=0.0$ and $Z / D=0.6$ of (a) mean static pressure, (b) RMS static pressure, (c) mean tangential velocity, (d) RMS tangential velocity, (e) mean axial velocity, and (f) RMS axial velocity, at $R e=0.87 \cdot 10^{5}, 1.74 \cdot 10^{5}, 2.80 \cdot 10^{5}, 3.48 \cdot 10^{5}$, and $4.35 \cdot 10^{5}$, respectively, inside Stairmand cyclone model. 

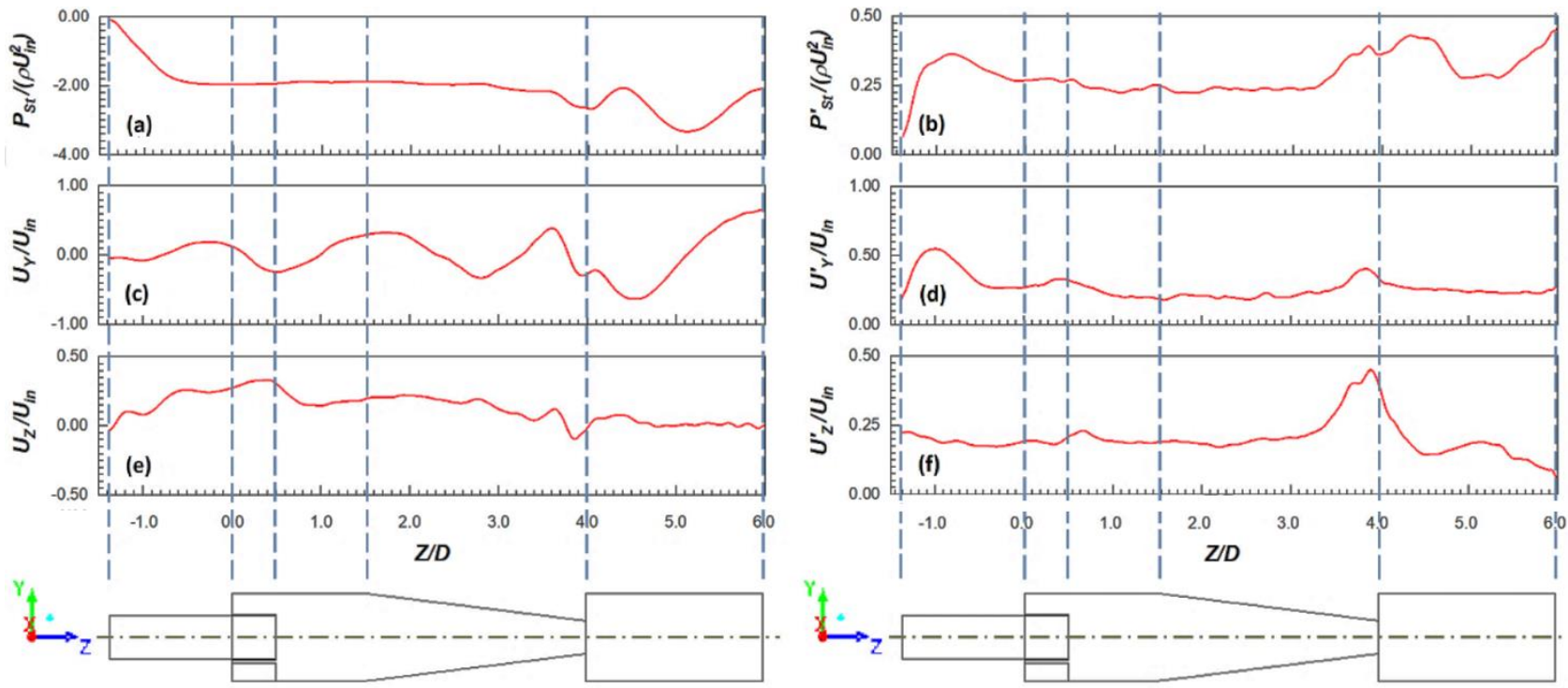

Fig. 12 The variations along the geometrical axis of Stairmand cyclone at $R e=2.80 \cdot 10^{5}$; from left to right: mean values and RMS values, from top to bottom: static pressure, tangential velocity and axial velocity, respectively. 